\title{
Maternidade segura no Brasil: o longo percurso para a efetivação de um direito
}

I 1 Lenice Gnocchi da Costa Reis, ${ }^{2}$ Vera Lucia Edais Pepe,

${ }^{3}$ Rosângela Caetano I

Resumo: A mortalidade materna é uma questão complexa e permanece sendo um grave problema de saúde pública. Este artigo se propõe a olhar esse fenômeno sob a ótica do direito à saúde, destacando que a opção por trazer a discussão para o campo dos direitos humanos proporcionou a ampliação de sua explicação e uma apresentação mais clara à sociedade. É apresentada breve trajetória da inserção desse tema na agenda política global, explorando algumas iniciativas internacionais e nacionais. $\mathrm{O}$ quadro atual da mortalidade materna no Brasil é apenas a face mais visível da precariedade da atenção à saúde das mulheres. A situação se agrava quando são considerados os casos de morbidade materna grave, que afeta um expressivo número de mulheres. Apesar das iniciativas para a efetivação do direito à maternidade segura, ainda são poucos os avanços, e a sociedade deve exigir que o Estado reverta o quadro de injustiça contra as mulheres.

> Palavras-chave: mortalidade materna, morbidade materna grave, direitos humanos.

\author{
${ }^{1}$ Médica sanitarista, mestre em \\ Ciências da Saúde, doutoranda \\ em Saúde Pública (ENSP), \\ pesquisadora do Departamento \\ de Administração e \\ Planejamento em Saúde, \\ ENSP. Endereço eletrônico: \\ lenicereis@ensp.fiocruz.br \\ ${ }^{2}$ Médica sanitarista, doutora \\ em Saúde Pública (ENSP), \\ pesquisadora do Departamento \\ de Administração e \\ Planejamento em Saúde, \\ ENSP. Endereço eletrônico: \\ verapepe@ensp.fiocruz.br \\ ${ }^{3}$ Médica, doutora em Saúde \\ Coletiva, professora adjunta do \\ Departamento de Planejamento \\ e Politicas Públicas do IMS- \\ UERJ. Endereço eletrônico: \\ caetano.r@gmail.com
}

Recebido em: 17/07/2010 Aprovado em: 21/04/2011 
A Organização Mundial da Saúde (OMS) afirma que a mortalidade materna permite identificar o lugar que a mulher ocupa na sociedade e reflete a adequação do sistema de saúde para dar respostas às suas necessidades (WHO, 1996). A morte materna revela, também, que o Estado negligencia seu dever de proteger a vida e a saúde da mulher, e que há na sociedade desigualdade entre gêneros, estando a mulher mais vulnerável (VENTURA, 2008). Nesse sentido, atualmente, há maior reconhecimento de que a redução da mortalidade materna não se restringe a uma questão de desenvolvimento; está relacionada à cidadania, aos direitos da cidadania e, sobretudo, deve ser vista como uma questão de direitos humanos.

Mann (1997) sugeriu apreciar as questôes relacionadas à saúde pela lente dos direitos humanos como contrapartida a outras perspectivas tal como a dos economistas que as veem sob o prisma da economia globalizada. Os direitos humanos fornecem um ponto de vista complementar, não restringindo essas questôes apenas a uma questão da saúde. Essa é uma linguagem que os Estados compreendem, e traz para o debate questôes como impunidade, responsabilidade, transparência e responsabilização (CHINKIN, 2006).

Os direitos humanos oferecem arcabouço legal importante para orientação das ações de saúde pública, reforçando a responsabilidade dos governos. Bobbio (1992) salienta que o problema atual não é justificar os direitos humanos, mas como protegê-los. Portanto, não se trata de uma questão filosófica, mas política.

A garantia do direito à maternidade segura segue sendo um problema relevante e a morte materna atinge milhares de mulheres anualmente. Apesar das iniciativas empreendidas na última década, poucos avanços foram observados. Isso também ocorre no Brasil, onde a redução nas taxas de mortalidade materna, nos últimos anos, não foi significativa. A razão de mortalidade materna, em 2006, foi de 77,2/100 mil nascidos vivos, com predomínio das causas obstétricas diretas: transtornos hipertensivos, com destaque para pré-eclâmpsia e eclâmpsia; complicações hemorrágicas e complicações do parto e do trabalho de parto (BRASIL, 2006).

Essa é apenas uma parte do problema. Atualmente, vêm sendo publicados trabalhos que evidenciam a ocorrência de casos de morbidade materna grave (near miss materno). Grande parte desses eventos poderia ser evitada e sabe-se que estão relacionados à qualidade da atenção obstétrica oferecida à população. Entretanto, ainda são poucos os estudos realizados no país sobre esse tema. 
Este trabalho se propõe a olhar esses eventos - a morte materna e o near

miss materno - sob a ótica do direito à saúde, explorando algumas iniciativas internacionais e nacionais para a minimização desse problema de saúde.

\section{Maternidade segura como parte dos direitos humanos e do direito das mulheres}

O conceito de cidadania carrega uma ideia fortemente relacionada aos direitos à igualdade e à liberdade. Os direitos de cidadania não estão necessariamente vinculados a valores universais, mas a decisões políticas. Não são direitos universais, refletem a organização do Estado e as forças que atuam em cada sociedade (BARSTED, 2005). No entanto, em muitos casos, os direitos do cidadão coincidem com os direitos humanos, que são mais abrangentes. São considerados direitos naturais, porque dizem respeito à dignidade da natureza humana e prescindem de qualquer lei para serem exigidos. Apesar de universais e naturais, são também históricos, produto da civilização humana e, como tal, susceptíveis a transformações em função de novas necessidades e avanços tecnológicos (BOBBIO, 1992). Se, em um primeiro momento, afirmaramse como direitos de liberdade, limitando o poder do Estado, no segundo momento foram propugnados os direitos políticos, que ampliaram o conceito de liberdade para além do não-impedimento, mas como autonomia. Por fim, foram proclamados os direitos sociais, frutos do amadurecimento de novos valores, como bem-estar e igualdade.

Os direitos humanos são legalmente garantidos pelas constituições, leis nacionais e por tratados regionais e internacionais, visam proteger os indivíduos e grupos contra ações e interesses que interferem em suas liberdades fundamentais e na dignidade humana (ONU, 1948).

Após a aprovação da Declaração Universal dos Direitos Humanos, em 1948, seus princípios foram traduzidos em tratados. Entre eles, figura a Convenção Internacional sobre a Eliminação de todas as Formas de Discriminação contra a Mulher, de 1979, que estabelece que todos os Estados signatários têm o dever de adotar medidas que eliminem a discriminação contra as mulheres e garantam a igualdade de condições entre mulheres e homens, inclusive em relação ao acesso a serviços de saúde. O cumprimento da Convenção é monitorado pelo Comitê para a Eliminação de todas as Formas de Discriminação contra a Mulher (CEDAW) 
e sua Recomendação Geral no. 24 postula que negligenciar o acesso a serviços de saúde necessários somente às mulheres é uma forma de discriminação (CEDAW, 2007). Portanto, serviços obstétricos com baixo padrão de qualidade e a falta de leitos obstétricos ou sua distribuição inadequada, conforme já apontado por Diniz (1996), constituem violação ao direito das mulheres à equidade e à assistência.

A iniciativa de discutir a mortalidade materna no campo dos direitos humanos proporcionou a ampliação da explicação e uma apresentação mais clara à sociedade. A partir dessa nova abordagem, fica evidente que a intervenção sobre esse fenômeno social complexo requer uma multiplicidade de visões e uma coalizão de forças para que o Estado cumpra seu dever de proteção à vida e garanta tratamento digno para todos.

Apesar dos avanços na área da saúde, da volumosa produção científica e da incorporação intensiva de tecnologias, ainda hoje nos deparamos com a morte materna. É um evento dramático, que traz repercussões danosas às famílias, aos recém-natos, que necessitam dos cuidados maternos, e ativa o ciclo da pobreza na sociedade. Nos serviços de saúde, gera angústia e tensão entre os membros das equipes e pacientes. Pelo simples fato de engravidar, processo natural da reprodução humana, a mulher assume uma carga de risco que tem sido negligenciada, pois a maior parte dessas mortes poderia ser evitada (CECATTI et al., 1999).

Essa morte é um evento sentinela, conceito cunhado por Rutstein et al. (1976), pois há tecnologia médica efetiva para evitá-la. Sua ocorrência alerta para falhas no cuidado prestado e deve deflagrar medidas para que suas causas sejam esclarecidas. É, portanto, importante marcador da qualidade do sistema de saúde, em especial em relação ao acesso, à adequação e à oportunidade do cuidado (BENAGIANO; THOMAS, 2003).

Esse tema permanece como desafio para pesquisadores, gestores e profissionais da área da saúde, por seu potencial de dano à sociedade, caráter de injustiça social e de violação dos direitos reprodutivos e, por conseguinte, dos humanos (COOK et al., 2004). Permanece, ainda, dada a escassez de informação sobre diversos aspectos.

Alguns autores têm alertado para o fato de que a morte materna é apenas a ponta de um iceberg (LAURENTTI, 1988). Recentemente, tem havido ênfase no estudo da morbidade materna grave, situação gravíssima em que não se chega ao óbito. Trata-se de uma situação muito mais comum que a morte 
materna, e que pode causar danos e prejuízos para a sociedade (CALLAGHAN

et al., 2008). Por ser mais frequente que a morte, recomenda-se que esse evento seja investigado, pois permitiria análise mais precisa dos fatores relacionados à sua ocorrência (SOUZA et al., 2006).

Cumpre destacar que tanto a morte quanto a morbidade materna grave não se distribuem de modo aleatório entre as mulheres. Revelam a iniquidade da sociedade, pois nem todos usufruem dos avanços e conhecimentos na medida de sua necessidade (TANAKA, 1995). Os estudos mostram que se concentram nos países em desenvolvimento, afetam as mulheres de menor renda, menor escolaridade e as negras (WHO, 2004).

É fundamental que o problema seja compreendido a partir de diferentes abordagens e ênfases, para possibilitar propostas de intervenções adequadas para a efetivação do direito a cuidados de qualidade à gestação, parto e puerpério e, consequentemente, para a redução de mortes e danos desnecessários.

\section{A mortalidade materna na agenda política internacional}

A Convenção sobre a Eliminação de todas as Formas de Discriminação contra a Mulher, citada anteriormente, é um marco importante na luta contra a mortalidade materna. A partir daí, a discriminação contra as mulheres passa a ser reconhecida como violação do princípio da igualdade de direitos e do respeito à dignidade humana, e também como impedimento da participação da mulher na vida da sociedade em condições iguais às do homem. Passam a ser estimuladas ações afirmativas para eliminar essa discriminação e impulsionar a igualdade. É de particular interesse o artigo $12^{\circ}$ da Convenção, que estabeleceu que os Estados-Membros deveriam adotar medidas para eliminar a discriminação contra as mulheres, no tocante aos cuidados à saúde, bem como garantir assistência apropriada durante todo o ciclo gravídico-puerperal (UNITED UNION, 1979).

A Conferência sobre "Maternidade Segura", realizada em Nairóbi, em 1987, foi fruto do reconhecimento internacional da morte materna como tragédia que necessitava ser mundialmente enfrentada (STARRS, 1987). A Iniciativa Mundial para a Maternidade Segura, lançada nessa ocasião, cuja meta era reduzir em 50\%, até o ano 2000, a mortalidade materna observada em 1985 (MAHLER, 1987), foi reiterada em outros encontros de caráter global. Reconhecia-se que a morte materna estava relacionada a uma desvantagem 
social e, portanto, precisaria incorporar intervenções de diversas naturezas (WHO; UNFPA; UNICEF; WORLD BANK, 1999).

Três anos depois, foi lançado o Plano de Ação Regional para a Redução da Mortalidade Materna, da Organização Pan-americana de Saúde (OPS/OMS, 1990). Em seguida, a Conferência Internacional das Nações Unidas para a População e Desenvolvimento foi realizada no Cairo, e gerou um documento contendo iniciativas voltadas para o desenvolvimento humano - o Programa do Cairo. Nesse documento, foi cunhada a definição de "saúde reprodutiva", reconhecendo-se que implicava, entre outras questôes, o direito de acesso a serviços e cuidados de saúde adequados, para garantir gravidez e parto seguros (UNITED NATIONS, 1994).

A desigualdade em relação à atenção à saúde foi um dos principais temas da $4^{\mathrm{a}}$ Conferência Mundial sobre as Mulheres, realizada em Beijing, em 1995. Afirmou-se o direito da mulher de desfrutar o mais elevado nível de saúde e de ter o controle sobre a própria vida reprodutiva e sobre a fertilidade. Recomendouse o compartilhamento da responsabilidade reprodutiva com o homem, o que poderia significar melhoria na saúde da mulher (UNITED NATIONS, 1995a).

Apesar desses esforços, ao final da década de 1990, as taxas de mortalidade materna não haviam decrescido. A pergunta que se fazia ainda era a mesma que William Farr fizera em 1838, na Inglaterra: por quanto tempo ainda perduraria esse sacrifício? (MAINE; ROSENFIELD, 1999).

Em 2000, a ONU lançou a Declaração do Milênio, e entre seus oito grandes objetivos para com o desenvolvimento humano, está melhorar a saúde materna (UNITED NATIONS, 2000). Apesar do consenso acerca da injustiça da morte materna, vista como privação dos direitos humanos das mulheres, o relatório de avaliação dos objetivos do milênio, de 2008, aponta que esse foi o objetivo de menor progresso e a morte materna segue atingindo milhares de mulheres anualmente (UNITED NATIONS, 2008).

Para entender a inserção da mortalidade materna na agenda política global e os resultados tímidos alcançados, Shiffman e Smith (2007) analisaram essa questão e observaram que houve dificuldades na obtenção de apoio político global. O primeiro elemento considerado na análise foi o comportamento dos atores políticos. Identificaram fragmentação do poder e falta de liderança. As vítimas são mulheres pobres que vivem em países em desenvolvimento e detêm pouco poder político. O segundo elemento foi o conjunto de ideias a respeito do 
tema: como o problema é reconhecido, apresentado à sociedade e as soluções

possíveis. Concluíram que não houve compreensão da questão e clareza das intervenções. Em relação ao contexto político, consideraram que foram abertas janelas de oportunidade na política global, mas não conseguiram observar como foram aproveitadas, ressaltando a fragmentação da estrutura global de governança na área da saúde, que não provê uma plataforma para ação coletiva mais efetiva. $\mathrm{O}$ último elemento analisado diz respeito às características do problema em si. Alertaram que sua gravidade não pode ser evidenciada pelos níveis de mortalidade, porque são baixos quando comparados aos de outras condições que competem por recursos. Sobre isso, Cook et al.(2004) salientam que a maternidade não é uma doença e, portanto, não deve ser comparada com outros problemas de saúde. Chamaram atenção para o fato de que a medição da mortalidade materna persiste como um problema, sem consenso acerca da maneira mais adequada de monitorar a situação. Também não há consenso sobre quais as intervenções mais efetivas e que devem ser priorizadas. Concluíram que todas essas dificuldades acabaram por prejudicar a iniciativa global para a maternidade segura.

\section{Mortalidade materna e morbidade materna grave: breve panorama da situação}

A morte materna, vale lembrar, é aquela que ocorre durante a gestação ou até 42 dias após seu término. Deve-se a qualquer causa relacionada à ou agravada pela gravidez, exceto as causas acidentais ou incidentais. Pode ser dividida em três grupos de causas: obstétricas diretas, obstétricas indiretas e não obstétricas. $\mathrm{O}$ primeiro grupo está relacionado com complicaçōes obstétricas na gravidez, parto ou puerpério, decorrentes de intervenções, omissões ou tratamento incorreto. $\mathrm{O}$ segundo é composto por óbitos que resultam de doenças prévias à gravidez ou que se desenvolvem nesse período, agravadas pelos efeitos fisiológicos da gravidez. O terceiro são aqueles óbitos por causas acidentais ou incidentais. (OMS, 1995).

A evolução da situação da mortalidade materna é de difícil acompanhamento. Houve mudanças nos métodos para estimar seu número e persistem as dificuldades relacionadas à disponibilidade de dados fidedignos (SHAH; SAY, 2007). Adotar um indicador comum é importante para que se possam estabelecer comparaçōes ao longo do tempo e entre lugares diferentes. Para dimensionar o quadro da mortalidade materna, o indicador mais utilizado é a razão de mortalidade materna (RMM), tido 
como uma medida de risco de morrer uma vez que se está grávida, que considera o número de óbitos maternos por 100 mil nascidos vivos (WHO, 2004).

O Brasil vem apresentando cifras superiores a 70 óbitos maternos/100.000 nascidos vivos $(\mathrm{NV})$. Alcançar o padrão aceitável estabelecido pela OMS entre 10 e 20 óbitos maternos/100.000 NV (WHO/UNICEF, 1996) - parece ser um desafio que exigirá maior empenho.

Em estudo do Ministério da Saúde (BRASIL, 2006), foi observado que, para o conjunto das capitais brasileiras, $56,5 \%$ dos óbitos por causas maternas foram por causas obstétricas diretas, com predomínio dos transtornos hipertensivos. As complicações relacionadas ao parto e ao trabalho de parto, e as hemorragias, também foram causas importantes, sendo responsáveis por 10,4\% e $9 \%$ do total de óbitos, respectivamente. Como a mortalidade materna tem sido utilizada como uma medida para monitorar a qualidade dos serviços de saúde (MINKAUSKIEN et al., 2004), pode-se inferir, pelos dados acima, que há graves problemas na qualidade da atenção a todo ciclo gravídico-puerperal.

A análise dos óbitos maternos constitui-se em fonte rica para o reconhecimento dos fatores envolvidos na sua ocorrência, porém pode ser insuficiente para identificar as falhas dos serviços de saúde. Embora a morte materna seja um grave problema de saúde pública, nas últimas décadas, tornou-se, em números absolutos, um evento raro, sobretudo considerando sua ocorrência por unidade de saúde e, quando utilizado de forma isolada, sua sensibilidade diminui como marcador da qualidade dos serviços (PENNEY; BRACE, 2007). A partir dessa constatação, houve um crescente interesse sobre o estudo da morbidade materna grave.

Stones et al. (1991) identificaram a ocorrência de morbidade materna grave, ou seja, episódios em que havia ameaça à vida da paciente mas que não evoluíram para óbito, por adequada intervenção médica ou, mesmo, por acaso. Esses casos foram denominados como near miss materno ou "quase morte" materna. O conceito é carregado de tensão, pois se uma vida foi salva é porque foi, de alguma forma, seriamente ameaçada. Foi sugerido que sua revisão poderia ser complementar à revisão dos óbitos maternos - pois ambos têm características semelhantes, sendo a "quase morte" mais frequente - o que permitiria aprofundar a avaliação da qualidade do cuidado obstétrico hospitalar e identificar as falhas do processo de atenção. A partir desse estudo, a morbidade materna grave vem chamando a atenção, com produção científica crescente (SOUZA et al., 2006). 
Em Campinas, foi realizado um estudo em que foram analisados dados de

2.207 mulheres que tiveram parto entre outubro/2005 e julho/2006, e observada uma razão de morbidade grave de 6,8/1.000 partos (LUZ et al., 2008).

Em Recife, outro estudo analisou casos considerados near miss admitidos em uma Unidade de Terapia Intensiva obstétrica. As causas de admissão mais comuns foram síndromes hipertensivas, hemorragia e infecção, e o parto foi cesáreo em $68,4 \%$ das pacientes. Os autores advogam que o modelo proposto por Thaddeus e Maine (1994), que relaciona a mortalidade materna com ocorrência de atraso na decisão de buscar assistência médica, no acesso da paciente ao serviço médico e em receber o tratamento adequado ("modelo dos três atrasos"), também se aplica para explicar parte dos casos de near miss (AMORIM et al., 2008).

Um grupo de pesquisadores brasileiros dedicados ao tema levanta algumas hipóteses acerca das sobreviventes dos casos classificados como near miss. Consideram que há maior probabilidade de que a qualidade de vida dessas mulheres seja afetada por stress pós-traumático, depressão pós-parto e comprometimento da vida sexual. As crianças também seriam afetadas desfavoravelmente, com piores resultados perinatais, maior ocorrência de danos neurológicos e menor ganho de peso (CECATTI, 2009).

A auditoria dos casos de morbidade materna grave vem sofrendo sugestões de ajustes. Recentemente, a OMS iniciou um processo para propor procedimentos que a tornem mais operacional; espera-se que seus resultados ganhem consistência e permitam maior comparabilidade, o que pode ajudar na melhoria da qualidade da assistência nos serviços obstétricos (SAY et al., 2009).

\section{A mortalidade materna e os direitos humanos: iniciativas do governo brasileiro}

O Brasil é signatário de vários acordos, tratados e convenções internacionais relacionados à questão da maternidade, assumindo compromissos que podem ser objeto de acompanhamento e cobrança. Ao serem ratificados pelo Poder Legislativo, esses instrumentos ganham força de lei e o não cumprimento, por parte das autoridades públicas, implica crime de responsabilidade. Desde a década de 1980, as questôes ligadas à saúde da mulher têm ganhado espaço na agenda política do país. O lançamento, pelo Ministério da Saúde, do Programa de Assistência Integral à Saúde Mulher (PAISM), a constituição de Comitês de 
Mortalidade Materna e a criação do Conselho Nacional dos Direitos da Mulher (CNDM) são fatos marcantes desse período.

O PAISM incorporou princípios e diretrizes que vinham sendo discutidos pelo Movimento Sanitário como base para o Sistema Único de Saúde (SUS). Pretendia oferecer às mulheres um conjunto de ações com amplo acesso e esperava obter forte impacto na mortalidade e morbidade dessa população (Brasil, 1984). Para Osis (1998), o PAISM significou um avanço para o reconhecimento dos direitos reprodutivos, embora não tenha sido implementado em todo o país, pela falta de compromisso político.

A constituição dos comitês estaduais de morte materna foi outra estratégia do Ministério da Saúde delineada nessa época. São de natureza interinstitucional e multiprofissional e se propõem a analisar todos os óbitos maternos, guardando confidencialidade, mas apontando medidas de intervenção para sua prevenção. Essa iniciativa chegou ao Brasil com mais de cinquenta anos de atraso, pois já havia sido adotada pelos Estados Unidos desde 1931 (COOK, 1989). Em 2005, o Brasil contava com: 27 comitês estaduais, 172 regionais, 748 municipais e 206 hospitalares (BRASIL, 2007).

O CNDM, criado em 1985 e, inicialmente, vinculado ao Ministério da Justiça, tinha por objetivo promover políticas para eliminar a discriminação contra a mulher e assegurar sua participação nas diversas esferas da vida da sociedade. A partir de 2003, tornou-se um órgão colegiado, de caráter consultivo da Secretaria Especial de Políticas para as Mulheres da Presidência da República. Foi criada também a Comissão Nacional de Morte Materna, em 1994, composta por representantes de organizações governamentais, sociedades científicas, movimentos de mulheres e técnicos da área. A comissão tinha como atribuição elaborar diagnóstico da mortalidade materna no país e propor diretrizes para sua diminuição (BRASIL, 2007).

Com o movimento da descentralização do SUS, ao final da década de 1990, o município tornou-se responsável pelas atividades de vigilância epidemiológica da mortalidade infantil e materna, passo importante para reduzir a subenumeração de casos, agilizar a investigação e melhorar a qualidade dos dados (BRASIL, 1999).

Apesar das iniciativas desse período, até o ano 2000 a realidade não havia se alterado. A meta de redução da mortalidade materna proposta na Conferência de Nairóbi não foi alcançada pelo país (TANAKA, 2001). Teve início, então, um 
período de intensa produção de portarias e criação de programas. O Ministério da

Saúde lançou, em 2000, o Programa de Humanização no Pré-natal e Nascimento (PHPN). Seu objetivo era ampliar o acesso e a cobertura dos serviços de atenção ao pré-natal, parto e puerpério e melhorar sua qualidade. Entre suas diretrizes, salientam-se duas: o reconhecimento do direito da gestante de saber onde será atendida no momento do parto, com seu acesso assegurado; e, ter uma assistência prestada de forma humanizada e segura (BRASIL, 2000a). Na mesma época, foi publicada uma portaria (BRASIL, 2000b) que objetivava melhorar a qualidade do pré-natal, afirmando a necessidade de classificação do risco gestacional e de garantir o atendimento em unidades de referência para gestaçôes de alto risco.

Em 2003, o óbito materno passou a ser considerado evento de notificação compulsória, tornando obrigatória sua investigação por parte dos municípios (BRASIL, 2003). Mas somente em 2008 a investigação do óbito materno foi regulamentada, atribuindo-se aos Núcleos Hospitalares de Epidemiologia a tarefa de busca ativa de casos, com prazos para o encerramento da investigação epidemiológica e disponibilização de dados no Sistema de Informaçôes de Mortalidade (BRASIL, 2008).

A iniciativa mais incisiva para o enfrentamento da mortalidade materna foi o lançamento do Pacto Nacional pela Redução da Mortalidade Materna e Neonatal. Aprovado em 2004 pela Comissão Intergestores Tripartite, instância de pactuação dos gestores do SUS das três esferas de governo, com a meta de reduzir em 15\% os índices de mortalidade materna e neonatal até 2006 e, em 75\%, até 2015 (BRASIL, 2004). Foram selecionados 78 municípios para serem apoiados financeiramente, todos com mais de 100.000 habitantes, baixo Índice de Desenvolvimento Humano e altas taxas de mortalidade materna e neonatal.

$\mathrm{O}$ pacto tem entre seus princípios norteadores a decisão política de investimentos na melhoria da atenção obstétrica e neonatal, e o respeito aos direitos humanos de mulheres e crianças. É integrado por um conjunto de açôes estratégicas para reorganizar o sistema, qualificar os serviços e humanizar a atenção. Para acompanhar o desenvolvimento das ações do pacto, foi criada a Comissão Nacional de Monitoramento e Avaliação do Pacto (BRASIL, 2005a).

Como frutos das discussões do pacto podem ser identificadas duas outras iniciativas: a publicação de lei que garante às parturientes o direito de ter um acompanhante durante sua internação (BRASIL, 2005b), e o lançamento da Política Nacional de Atenção Obstétrica e Neonatal (BRASIL, 2005c). 
O Pacto pela Saúde, de 2006, estabeleceu como ações prioritárias a melhoria da saúde materna e a redução da mortalidade infantil. Tornou-se obrigatória a inclusão dessas ações nos Planos Estaduais e Municipais de Saúde, firmando o compromisso dos gestores com o alcance de metas pactuadas (BRASIL, 2006).

Outra iniciativa relevante foi o lançamento da Campanha Nacional de Incentivo ao Parto Normal e Redução da Cesárea Desnecessária, em 2008. Desde o início da década de 1990, Faúndes e Cecatti (1991) já apontavam que nenhum outro país apresentava uma curva de aumento da taxa de cesáreas tão acentuada, nem taxas em níveis tão altos, como o Brasil.

No campo normativo, cabe ainda mencionar (I) a publicação de lei que estabelece a vinculação do pré-natal ao local de parto, afirmando como direito da gestante conhecer a maternidade onde receberá assistência, no âmbito do SUS (BRASIL, 2007b), e (II) o estabelecimento de parâmetros para o funcionamento dos serviços de atenção obstétrica e neonatal (BRASIL, 2008).

No âmbito da pesquisa, o Ministério da Saúde empenhou-se em inserir na Agenda Nacional de Prioridades de Pesquisa em Saúde, publicada em 2005, a subagenda da Saúde da Mulher, agregando vários temas (BRASIL, 2005d).

Em 2007, ao analisar os relatórios encaminhados pelo governo brasileiro e pela sociedade civil brasileira, o CEDAW fez elogios pelos avanços demonstrados, em especial pela lei sobre violência doméstica (Lei Maria da Penha), mas fez recomendaçôes para o efetivo cumprimento dos direitos previstos na Convenção. Mostrou preocupação com o fato de a mortalidade materna permanecer elevada, com a magnitude da gravidez na adolescência e com o número elevado de abortos realizados de forma não segura. Exortou o país a aumentar o acesso das mulheres à assistência à saúde, em particular aos serviços de saúde sexual e reprodutiva. Solicitou, ainda, que o Pacto Nacional pela Redução de Mortalidade Materna fosse objeto de monitoramento. Em relação à legislação sobre a criminalização do aborto, foi recomendada a revisão das disposições punitivas para as mulheres que passam por aborto, conforme Recomendação Geral no 24 (CEDAW, 2007).

O Ministério da Saúde (BRASIL, 2006) reconhece que alguns problemas persistem e precisam ser enfrentados. Entre eles: a investigação insuficiente dos óbitos maternos; os recursos financeiros insuficientes, com baixa execução e desvio dos objetivos de aplicação; a descontinuidade na gerência de programas;e a inadequação da formação e da gestão dos recursos humanos, com dificuldade para fixação de profissionais de saúde no interior do país. 


\section{Os serviços obstétricos: especificidades e desafios}

A gravidez e o parto são processos fisiológicos. Portanto, a maioria das mulheres que são atendidas nos serviços obstétricos necessita apenas de cuidados básicos. Contudo, na área obstétrica podem ocorrer emergências que costumam ser repentinas e que devem ter respostas rápidas. Também é sabido que a mortalidade materna pode ser extremamente alta nos primeiros dois dias após o parto (RONSMANS; GRAHAM, 2006). Portanto, ter acesso a serviços obstétricos de qualidade é fundamental para reduzir a mortalidade e a morbidade maternas.

O processo de atenção ao parto ganha especificidade por envolver duas vidas. Isso obriga o serviço de saúde a prover um conjunto de elementos para que tanto a mãe como o concepto desfrutem de um parto seguro. Além disso, algumas mudanças ocorridas na sociedade nas últimas décadas tiveram grande repercussão para os serviços obstétricos e trouxeram novas demandas que interferem na qualidade do cuidado e, sobretudo, na segurança dos pacientes. $\mathrm{Na}$ atualidade, observam-se o aumento tanto do número de mulheres que engravidam pela primeira vez em idade mais avançada como o de adolescentes grávidas; mulheres com patologias que, com os avanços na área médica, podem engravidar e levar a gravidez a termo; e, ainda, o aumento da obesidade e o maior uso de tratamentos de fertilidade. Esse conjunto tem trazido novas preocupações e o processo de atenção na área obstétrica torna-se mais complexo (MISRA; GRASON, 2006).

A realização do parto em serviços de saúde é reconhecida como medida sanitária relevante para a redução da morte materna (GAY et al., 2003). Mas isso, por si só, não significa que os partos estejam sendo atendidos de modo adequado. Ao analisar os dados coletados pela Pesquisa Assistência Médico-Sanitária, em 1999, Leal e Viacava (2002) apontaram que quase um terço dos partos do país ocorria em instituições que não tinham obstetras nem anestesistas, e mais de dois terços não contavam com a presença de pediatras.

Essa realidade não parece ter sido alterada, pois em 2004/2005 foi realizada pesquisa em 12 maternidades do interior do estado de São Paulo, e observou-se que o obstetra muitas vezes estava em regime de plantão à distância, e ainda que a área física onde ocorria o pré-parto era inadequada. Quanto à adoção de práticas efetivas para o manejo do parto, preconizadas pelo PHPN, como a presença de acompanhante e o controle não farmacológico da dor, observou-se que são pouco incorporadas, enquanto outras sabidamente prejudiciais, como a 
venóclise, a tricotomia e a episiotomia, são frequentemente utilizadas (PARADA; CARVALHAES, 2007). Resultados semelhantes já haviam sido apresentados por D'Orsi et al. (2005), que, ao avaliarem a qualidade da atenção durante o processo de trabalho de parto em duas maternidades, concluíram que a frequência de intervenções durante a assistência ao parto era elevada.

Em estudo mais recente, realizado em maternidades no estado do Rio de Janeiro, Magluta et al. (2009) verificaram que havia deficiências nas instalaçôes físicas, na disponibilidade de equipamentos, de instrumental cirúrgico e de material, e insuficiência no quadro de recursos humanos, demonstrando que a adequação da estrutura desses serviços ainda não foi equacionada. Embora, como há muito enfatizado por Donabedian (1980), se reconheça que a estrutura não determina os resultados, e que estes podem ser influenciados tanto pelas características dos serviços (estrutura e processo) como dos pacientes, é inegável que uma estrutura adequada favorece o desenvolvimento de processos mais adequados e resultados mais favoráveis.

$\mathrm{O}$ arsenal tecnológico hoje disponível propicia um alto grau de intervenção médica. Para Wennberg (2005) a oferta de recursos regula a frequência da sua utilização e, muitas vezes, se observa um uso abusivo,e é inegável que há um paradoxo nessa situação - quanto mais cuidado, maior exposição aos perigos potenciais (DAVIS, 2004). Johanson et al. (2002) apontam que há uma tendência crescente à medicalização do parto normal, inclusive com a incorporação de tecnologias sem efetividade comprovada. Os esforços para conhecer o processo de trabalho dos serviços obstétricos, as falhas e os problemas que ocorrem podem contribuir para a melhoria da qualidade da atenção e para a redução de riscos para mãe e filho.

\section{Considerações finais}

A morte e a morbidade maternas preveníveis podem ser vistas como resultado da violação dos direitos humanos - direito à vida, a viver livre de discriminação, a usufruir o mais alto padrão de saúde possível. Essa abordagem permite tornar explícito para a sociedade que a morte e a morbidade maternas não são fenômenos naturais, que se distribuem ao acaso. Permite também afirmar que o Estado tem obrigação de respeitar, proteger e prover esses e outros direitos.

O Brasil ratificou importantes tratados e pactos internacionais que tratam dos direitos humanos e da mulher. No entanto, essa adesão formal não garante 
que a saúde materna seja tomada como prioridade, ainda que o governo possa ser responsabilizado pelas falhas em garantir às mulheres o acesso à atenção de boa qualidade no momento oportuno, sem discriminação, sem importar se o sistema de saúde é centralizado ou descentralizado, se público ou privado.

Nas últimas décadas, foram muitas as iniciativas globais para enfrentar a mortalidade materna. Seus resultados, no entanto, ainda são acanhados. No Brasil, também se observam esforços, em especial de caráter normativo, com alcance limitado. A meta proposta pela Declaração do Milênio permanece como desafio, e a lista de recomendaçôes para reverter o quadro da saúde materna no país é extensa, pois representa desigualdades e injustiças acumuladas ao longo de sua história.

Dentre as intervenções para reduzir a mortalidade materna, promover a melhoria da qualidade dos serviços obstétricos reveste-se de singular importância. Mas além de tornar as tecnologias, sabidamente custo-efetivas, disponíveis para todos que delas necessitem, é importante aprofundar o conhecimento acerca dos resultados de sua utilização e identificar problemas que podem contribuir para gerar resultados negativos.

O SUS deve ser capaz de mobilizar líderes profissionais e da sociedade, coordenar as ações, monitorar a implementação das políticas públicas voltadas para esse tema e prestar contas à sociedade. A sociedade também precisa estar atenta à alocação e destinação dos recursos públicos, exigir o cumprimento das leis, normas, regulamentos, metas programáticas e a inclusão dessa questão como prioridade na agenda política nacional.

\section{Colaboradores}

As autoras participaram igualmente na elaboração e discussão do artigo.

\section{Referências}

AMORIM, M.M.R.; KATZ, L.; VALENÇA, M.; ARAUJO, D.E. Morbidade materna grave em UTI obstétrica no Recife, regiāo nordeste do Brasil. Rev. Assoc. Med. Bras., v.54, n.3, p.261-266, 2008.

BARSTED, L.L. Novas legalidades e novos sujeitos de direitos. In: AVILA, M.B.; PORTELlA, A.P.; FERREIRA, V. (Org.). Novas legalidades e democratização da vida social: familia, sexualidade e aborto. Rio de Janeiro: Garamond, 2005. p.29-38. 
BENAGIANO, G.; THOMAS, B. Safe motherhood: the FIGO initiative. Int J Gynaecol. Obstet., v.82, n.3, p.263-74, 2003.

BOBBIO, N. A era dos direitos. Rio de Janeiro: Campus, 1992.

BRASIL. Ministério da Saúde. Pacto nacional pela redução da mortalidade materna e neonatal. Brasília: Ministério da Saúde; 2004.

. Secretaria de Atenção à Saúde. Departamento de Ações Programáticas Estratégicas. Estudo da mortalidade de mulheres de 10 a 49 anos, com ênfase na mortalidade materna: relatório final. Brasília: Ministério da Saúde, 2006.

- Assistência integral à saúde da mulher: bases da ação programática. Brasília: Ministério da Saúde, 1984.

. Secretaria de Atenção à Saúde. Departamento de Ações Programáticas Estratégicas. Manual dos comitês de mortalidade materna. Brasília: Ministério da Saúde, 2007.

. Secretaria de Ciência, Tecnologia e Insumos Estratégicos. Departamento de Ciência e Tecnologia. Agenda nacional de prioridades de pesquisa em saúde. Brasília: Ministério da Saúde, 2005d.

. Lei n.11.634,de 27 de dezembro de 2007. Dispóe sobre o direito da gestante ao conhecimento e a vinculação à maternidade onde receberá assistência no âmbito do Sistema Único de Saúde. Diário Oficial da União, Brasília, DF, 28 dez. 2007 b.

. Lei n.11.108, de 07 de abril de 2005. Altera a Lei 8.080, introduzindo o direito ao acompanhante de escolha durante o trabalho de parto, no parto e no pós-parto imediato. Diário Oficial da União, Brasília, DF, 8 abr. 2005 b.

- Portaria GM/MS n. ${ }^{\circ} 1.399$, de 15 de dezembro de 1999. Regulamenta a NOB SUS 01/96 e dá outras providências. Diário Oficial da Uniāo, Brasília, DF, 16 dez. 1999.

- Portaria GM/MS n.569, de 01 de junho de 2000. Institui o Programa de Humanização no Pré-natal e Nascimento no âmbito do Sistema Único de Saúde. Diário Oficial da União, Brasília, DF, 18 ago. 2000a.

. Portaria GM/MS no 570, de 01 de junho de 2000. Institui o Componente I do Programa de Humanização no Pré-natal e Nascimento. Diário Oficial da União, Brasília, DF, 18 ago. 2000 b.

. Portaria GM/MS n.o 653, de 28 de maio de 2003. Estabelece que o óbito materno passe a ser considerado evento de notificação compulsória. Diário Oficial da União, Brasília, DF, 30 mai. 2003.

Portaria GM/MS n. ${ }^{\circ}$ 427, de 22 de março de 2005. Institui a Comissão Nacional de Monitoramento e Avaliação da Implementação do Pacto Nacional pela Redução da Mortalidade Materna e Neonatal e dá outras providências. Diário Oficial da União, Brasília, DF, 23 mar. 2005a. 
. Portaria GM/MS n.o 1.067, de 4 de julho de 2005. Institui a Política Nacional de

Atenção Obstétrica e Neonatal, e dá outra providências. Diário Oficial da União, Brasília, DF, 06 jul. 2005c.

. Portaria GM/MS n.o 399, de 22 de fevereiro de 2006. Divulga o Pacto pela Saúde 2006 - Consolidação do SUS e aprova as Diretrizes Operacionais do Referido Pacto. Diário Oficial da União, Brasília, DF, 23 fev. 2006.

AGÊNCIA NACIONAL DE VIGILÂNCIA SANITÁRIA - ANVISA. Resolução RDC no 36, de 03 de junho de 2008. Dispõe sobre Regulamento Técnico para Funcionamento dos Serviços de Atenção Obstétrica e Neonatal. Diário Oficial da União, Brasília, DF, 08 jul. 2008.

CALLAGHAN, W.M.; MACKAY, A.P.; BERG, C.J. Identification of severe maternal morbidity during delivery hospitalizations, United States, 1991-2003.Am. J. Obstet. Gynecol., v.199, n.2, p.133.e1-133.e8, 2008.

CECATTI, J.G.; FAÚNDES, A.; SURITA, F.G.C. Maternal mortality in Campinas: evolution, under-registration and avoidance. São Paulo Med J.,v.117, n.1, p.5-12, 1999.

CECATTI, J.G.; LAUDARI, C. Mortalidade Materna no Nordeste: da política à ação. Salvador, Bahia, 2004 Disponível em <http://www.bvs-sp.fsp.usp.br/tecom/docs/2005/ cec001.pdf\#page=31>. Acesso em: 21 dez. 2009 .

CECATTI, J.G. et al. Brazilian network for the surveillance of maternal potentially life threatening morbidity and maternal near-miss and a multidimensional evaluation of their long term consequences. Reprod. Health, v.6, p.15, 2009.

CHINKIN, C. Health and Human Rights Public Health.Public Health, v.120, Supplement 1 , p.52-59, 2006.

COMITÊ SOBRE A ELIMINAÇÃO DA DISCRIMINAÇÃO CONTRA AS MULHERES (CEDAW). Comentários finais do Comitê sobre a Eliminação da Discriminação contra as Mulheres: Brasil; 10 de agosto de 2007. Disponível em: <http://200.130.7.5/spmu/ portal_pr/eventos_internacionais/ONU/39\%20sessão\%20CEDAW/Recomendações\%20 CEDAW\%20-\%20português.pdf>. Acesso em: 24 jan. 2010.

COOK, R. The role of confidential enquiries in the reduction of maternal mortality and alternatives to this approach.Int. J. Gynaecol. Obstet., v.30, n.1, p.41-5, 1989.

COOK, R.; DICKENS, B.M.; FATHALLA, M.F. Saúde reprodutiva e direitos humanos: integrando medicina, ética e direito. Rio de Janeiro: Cepia \& Oxford; 2004.

DAVIS, P. Health care as a risk factor. Can. Med. Assoc. J., v.70, n.11, p.1688-9, 2004.

DINIZ, C.S.G. Assistência ao Parto e Relaçôes de Gênero: Elementos para uma Releitura Médico-Social. 1997. Dissertação (Mestrado em Medicina) - Faculdade de Medicina, Universidade de São Paulo, São Paulo, 1997. 
DONABEDIAN, A.The definition of quality: approaches to its assessment. Ann Arbor: Health Administration Press; 1980.

D'ORSI, E. et al. Qualidade da atenção ao parto em maternidades do Rio de Janeiro. Rev. Saúde Pública, v.39, n.4, p.645-654, 2005

FAUNDES, A.; CECATTI, J.G. A operação cesárea no Brasil: incidência, tendências, causas, consequências e propostas de ação. Cad. Saúde Pública, v.7, n.2, p.150-173, 1991.

GAY, J. et al. What works: a policy and program guide to the evidence on family planning, safe motherhood and STI/HIVIAIDS interventions. Module 1: Safe motherhood. Policy Project. Washington; 2003. Disponível em: <http://www.policyproject.com/pubs/generalreport/ SM_WhatWorksps2.pdf>. Acesso em: 18 dez. 2009.

JOHANSON, R.; NEWBURN, M.; MACFARLANE, A. Has the medicalization of childbirth gone too far? BMJ, v.324, n.7342, p.892-5, 2002.

LAURENTI, R. Marcos referenciais para estudos e investigações em mortalidade materna. Rev. Saúde Públ., v.22, n.6, p.507-12, 1988.

LEAL, M.C.; VIACAVA, F. Maternidades do Brasil. Radis-Comunic Saúde, n.2, p.8-26, 2002. LUZ, A.G.; TIAGO, D.B; SILVA, J.C.G.; AMARAL, E. Morbidade materna grave em um hospital universitário de referência municipal em Campinas. RevBras Ginecol. Obstet., v.30, n.6, p.281-6, 2008.

MAGLUTA, C.et al. Estrutura de maternidades do Sistema Único de Saúde do Rio de Janeiro: desafio à qualidade do cuidado à saúde. Rev. Bras. Saúde Mater. Infant., v.9, n.3, p: 319-329,2009.

MAHLER, H. The safe motherhood initiative: a call to action. Lancet, v.329, n.8534, p.668-70. 1987.

MAINE, D.; ROSENFIELD, A. The Safe Motherhood Initiative: Why Has It Stalled? Am. J. Public Health, v.89, n.4, p.480-2, 1999.

MANN, J. Medicine and public health, ethics and human rights.Hastings Cent Rep., v.27, n.3, p.6-13, 1997.

MINKAUSKIEN, M.; NADISAUSKIEN, R.; PADAIGA, Z.; MAKARI, S. Systematic review on the incidence and prevalence of severe maternal morbidity. Medicina (Kaunas), v.40, n.4, p.299-309, 2004.

MISRA, D.P.; GRASON, H. Achieving safe motherhood: Applying a life course and multiple determinants perinatal health framework in public health. Women's Health Issues, v.16, n.4, p.159-175, 2006.

ORGANIZAÇÃO DAS NAÇŌES UNIDAS (ONU). Declaração Universal dos Direitos Humanos. Nova York, 1948. 
ORGANIZAÇĀO MUNDIAL DA SAÚDE. Classificação Estatística Internacional de

Doenças e Problemas Relacionados à Saúde -10a Revisão. 2a ed. São Paulo: Editora da Universidade de São Paulo; 1995.

Promovendo a maternidade segura através dos direitos humanos. Rio de Janeiro:

Cepia; 2003.

ORGANIZACIÓN PANAMERICANADE LASALUD. ORGANIZACIÓN MUNDIAL

DE LA SALUD. Plan de Acción Regional de la Mortalidad Materna en las Américas. XXIII Conferencia Sanitaria Panamericana. Washington, D.C.: OPS/OMS, 1990.

OSIS, M.J.M.D. PAISM: um marco na abordagem da saúde reprodutiva no Brasil. Cad. Saúde Pública, v.14, suppl.1, p. S25-S32, 1998.

PARADA, C.M.G.L.; CARVALHAES, M.A.B.L. Avaliação da estrutura e processo da atenção ao parto: contribuição ao debate sobre desenvolvimento humano. Rev. Latino-Am Enfermagem, v.15, n. spe, p.792-798, 2007.

PENNEY G.; BRACE, V. Near miss audit in obstetrics.Curr.Opin.Obstet. Gynecol., v.19, n.2, p.145-50, 2007.

RONSMANS, C.; GRAHAM, W.J. Maternal mortality: who, when, where, and why. Lancet, v.368, n.9542, p.1189-200, 2006.

RUTSTEIN, D.D. et al. Measuring the quality of medical care. A clinical method. $N$ Engl JMed., v.294, n.11, p.582-8, 1976.

SAY, L.; SOUZA, J.P.; PATTINSON, R.C. Maternal near miss - towards a standard tool for monitoring quality of maternal health car e. Best Pract Res ClinObstetGynaecol, v.23, n.3, p.287-296, 2009.

SHAH, I.H.; SAY, L. Maternal Mortality and Maternity Care from 1990 to 2005: Uneven but Important Gains. Reprod Health Matters, v.5, n.30, p.17-27, 2007.

SHIFFMAN, J.; SMITH, S. Generation of political priority for global health initiatives: a framework and case study of maternal mortality. Lancet, v.370, n.9595, p.1370-9, 2007.

SOUZA, J.P. et al.Revisão sistemática sobre morbidade maternal near miss.Cad. SaúdePública, v.22, n.2, p.255-64, 2006.

STARRS, A. Preventing the tragedy of maternal deaths: a report on the International Safe Motherhood Conference, Nairobi, Kenya, February 1987. World Bank, 56 p., 1987.

STONES, W.; LIM, W.; AL-AZZAWI, F.; KELLY, M.An investigation of maternal morbidity with identification of life-threatening 'near miss' episodes. Health Trends, v.23, n.1, p.13-5, 1991.

TANAKA, A.C. (Org.). Saúde da mulher e direitos reprodutivos: dossiê mortalidade materna. São Paulo-SP: Rede Nacional Feminista de Saúde e Direitos Reprodutivos - 
Rede Saúde; 2001. Disponível em: http://www.redesaude.org.br/Homepage/Dossi\%EAs/ Dossi\%EA\%20Mortalidade\%20Materna.pdf. Acesso em:17 nov.2009.

TANAKA, A.C. Maternidade: dilema entre nascimento e morte. São Paulo: Hucitec; Rio de Janeiro: Abrasco, 1995.

THADDEUS, S.; MAINE, D. Too far to walk: maternal mortality in context. Soc. Sci. Med., v. 8, n. 38, p.1091-110, 1994.

UNITED NATIONS.Convention on the Elimination of All Forms of Discrimination against Women.1979. Disponível em: <http://www.un.org/womenwatch/daw/cedaw/text/ econvention.htm>. Acesso em: $12 \mathrm{dez} .2009$.

- Programme of Action of the International Conference on Population and

Development.1994. Disponível em: <http://www.un.org/popin/icpd/conference/offeng/ eng694.html>. Acesso em: 12 dez.2009.

. Report of the Fourth World Conference on Women Beijing, 4-15 September 1995.

United Nations, New York, 1996. Disponível em: <http://www.un.org/womenwatch/daw/ beijing/official.htm>. Acesso em: 12 dez.2009.

. RES/55/2, de 18 September 2000. United Nations Millennium Declaration, 2000.

Disponível em: <http://www.un.org/millennium/>. Acesso em: $12 \mathrm{dez} .2009$.

UNITED NATIONS. The Millennium Development Goals Report - 2008. New York, 2008. Disponível em<http://www.un.org/millenniumgoals/maternal.shtml>. Acesso em: 2 mar.2010.

VENTURA, M. A mortalidade materna: a persistente violação do direito de proteção da vida e autonomia feminina. Rev. Bioét., v.16, n.2, p.217-228, 2008.

WENNBERG, J.E. Variation in use of Medicare services among regions and selected academic medical centers: is more better? New York: Commonwealth Fund, 2005.

WORLD HEALTH ORGANIZATION.Maternal mortality in 2000: estimates developed by WHO, UNICEF and UNFPA. Geneva: WHO; 2004. Disponível em: <http://www. unfpa.org/upload/lib_pub_file/237_filename_mm2000.pdf>. Acesso em: 18 dez.2009.

.UNICEF.Revised 1990 estimates of Maternal Mortality. A new approach by WHO and UNICEF.WHO/FRH/MSM 96.11. UNICEF/PZN/96.1. Geneva: WHO/UNICEF, 1996.

.UNFPA.UNICEF. WORLD BANK. Reduction of maternal mortality: a joint WHO/ UNFPA/UNICEF/World Bank Statement. Geneva; World Health Organization, 1999. 
Safe motherhood in Brazil: the long road to the realization of a right

Maternal mortality is a complex issue and remains a serious public health problem. This paper aims to look at this phenomenon from the perspective of the right to health, noting that the option for bringing the discussion to the field of human rights provided an extension of its explanation and a clearer presentation to society. It presents brief history of the insertion of this theme in the global policy agenda, exploring national and international initiatives. The current situation of maternal mortality in Brazil is just the most visible face of the precariousness of the health care of women. The situation worsens when cases of severe maternal morbidity are considered, which affects a significant number of women. Despite the initiatives for the realization of the right to safe motherhood, there are few advances, and society must require the State to reverse the injustice against women.

> Key words: maternal mortality, severe maternal morbidity, human rights. 